\title{
On Mathematical Stability Analysis of Liquefaction Considering Soil-Water Coupling
}

\author{
Jian CHEN $^{1}$, Hideyuki O-TANI ${ }^{2}$, Muneo HORI ${ }^{3}$ \\ ${ }^{1}$ Member of JSCE, Ph.D., RIKEN Advanced Institute for Computational Science (Minatojima-minami-machi 7-1-26, \\ Chuo-ku, Kobe 650-0047, Japan) \\ E-mail: jchen@riken.jp \\ ${ }^{2}$ Member of JSCE, Ph.D., RIKEN Advanced Institute for Computational Science (Minatojima-minami-machi 7-1-26, \\ Chuo-ku, Kobe 650-0047, Japan) \\ ${ }^{3}$ Member of JSCE, Ph.D., Professor of Earthquake Research Institute, The University of Tokyo (Yayoi 1-1-1, \\ Bunkyo-ku, Tokyo 113-0032, Japan)
}

\begin{abstract}
We study the stability of the solutions of the governing equations for soil liquefaction, assuming perturbations in the form of plane wave and of spherical wave. We model the dilatancy effect of soil for stability analysis by setting relevant components of constitutive tensors. Theoretical analysis shows that perturbations in the form of plane wave are always stable without dilatancy and can be unstable when dilatancy ratios exceed a certain critical value. We derive the critical dilatancy ratio explicitly for the plane wave case. For perturbations in the form of spherical wave, numerical simulations reveal similar dependency of stability on dilatancy ratios. As the existence of unstable solution is confirmed, our analysis provides a new perspective on possible initiation of liquefaction: a transition from stable to unstable solutions of the governing equations.
\end{abstract}

Key Words: liquefaction, stability analysis, soil-water coupling

\section{Introduction}

Liquefaction refers to a change in ground behaviour from solid-like to fluid-like ${ }^{1), 2)}$. In its normal state, soil serves as a stable civil engineering material. Induced by strong ground motions in earthquakes, soil at some specific site may lose its strength and start to flow, causing damages to structures on or within the liquefied ground. To understand liquefaction, extensive experimental studies ${ }^{1)}$ and field observations ${ }^{3)}$ have carried out. It is now generally accepted that in the process of liquefaction the pore pressure increases with the weakening of the contact of soil particles.

From the mechanics viewpoint, liquefaction is induced by the coupling of soil particles and pore fluids. Such coupling can be modelled from a microscopic and a macroscopic approach. Microscopically, the interaction between soil particles is modelled and the pore fluid phase is either described by the NavierStokes equations ${ }^{4)}$ or by Darcy's empirical law ${ }^{5)}$. Macroscopically, soil is treated as a continuum and its dynamics are derived based Biot's two-phase-mixture theory ${ }^{6)}$. For better prediction purpose, advanced constitutive models, e.g. elasto-plastic models ${ }^{7)}$ and hypo-plastic models ${ }^{8)}$, have been developed for numerical analysis of liquefaction.
Though progresses have been made in numerical studies of liquefaction ${ }^{7,9)}$, the stability of the solutions of the governing equations for liquefaction has not yet been fully discussed. Stability analysis will help to understand the initiation of liquefaction, which is an unstable phenomenon. Such understanding is important to make reliable numerical simulations. If an unstable solution in simulation is caused by mathematical instability, it should not be mistaken as caused by numerical instability (due to numerical methods or numerical errors) and not hence be suppressed wrongly. From a mathematical viewpoint, a transition from a stable to an unstable solution of the governing equations can be regarded as a possible local liquefaction initiation. If unstable solution exists, numerical techniques such as introduction of small perturbations can be used to capture the unstable solutions. Such kind of perturbations are known as "numerical imperfection" for numerical analysis of buckling phenomena ${ }^{10)}$ to obtain unstable solutions.

In this paper, perturbations in the form of plane wave and of spherical wave will be considered for the stability analysis of the solutions of the governing equations, analytically and numerically. In the stability analysis, the dilatancy effect of soil will be taken into account. Various kinds of stress-dilatancy rela- 


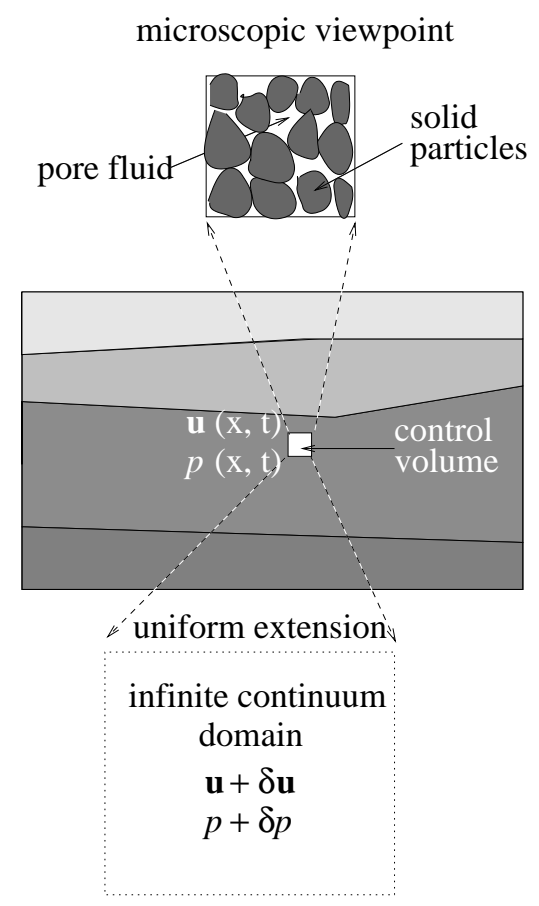

Fig.1 Continuum model of soil for stability analysis: From a microscopic viewpoint, soil consists of solid particles and pore fluids (water etc.); For continuum mechanics based modelling, a control volume of size much larger than particle size is considered with homogenized material properties and field variables ( $\mathbf{u}$ and $p$ ); Small perturbations of the field variables, $\delta \mathbf{u}$ and $\delta p$ are analysed in an infinite region as the extension of a small region.

tions have been proposed and used as the flow rules in plasticity models ${ }^{11)}$. It is seldom discussed that the influence of dilatancy on the stability of the solutions of the governing equations. In the present study, we propose a straightforward way to model the dilatancy effect for stability analysis.

This paper is structured as follows: the governing equations and the stability analysis are explained in the second section. The stability analysis for perturbations in the form of plane wave is discussed in the third section for non-dilatancy and dilatancy cases. Perturbations in the form of spherical wave are discussed in the fourth section, followed by concluding remarks and comments on future research.

\section{Formulation of the problem}

\section{(1) Governing Equations}

There are many ways to model the dynamics of soil. From a microscopic point of view, soil consists of solid particles, with pore space filled with water or other kinds of fluid, as shown in Fig. 1. Micromechanical models have been proposed for particleparticle interaction and particle-fluid interaction, e.g. a combination of discrete element modelling for solid particles and the averaged Navier-Stokes equations for pore water ${ }^{4}$. There are two main drawbacks of such micro-mechanical models: 1) It is difficulty to verify the models for interaction at particle level and 2) It is not feasible to model by particles for large systems (e.g. for a $1 \mathrm{~m} \times 1 \mathrm{~m} \times 1 \mathrm{~m}$ system with medium sand, of diameter $0.25 \mathrm{~mm}$ to $0.5 \mathrm{~mm}$, approximately $10^{10}$ to $10^{11}$ particles are needed). Thus macro-mechanical models based on continuum mechanics are more practical.

In this paper, we employ continuum mechanics based modelling for soil dynamics problems, assuming elasto-plasticity for soil and slow flow for water. More specifically, denoting by $\dot{\mathbf{u}}$, and $\dot{p}$ the rate of the displacement of soil and the rate of the pressure of water, we have

$$
\begin{array}{r}
\rho \mathrm{D}^{2} \dot{\mathbf{u}}-\nabla \cdot\left(\mathbf{c}^{e p}: \nabla \dot{\mathbf{u}}\right)+\nabla \dot{p}=\mathbf{0}, \\
\nabla \cdot \dot{\mathbf{u}}-\nabla \cdot(\mathbf{k} \cdot \nabla p)=0,
\end{array}
$$

where $\rho, \mathbf{c}^{e p}$ and $\mathbf{k}$ are soil density, elasto-plasticity and permeability; $\nabla$ and $\mathrm{D}$ stand for spatial and temporal derivatives; and ".", ":" stand for the first and second-order contraction of tensor.

Note that Eq. (1) is for the equilibrium (conservation of momentum) and the continuity (conservation of mass). The inertia and stress of soil and the pressure of water are in equilibrium, ignoring the body force due to gravity for simplicity. The divergence of the volumetric strain rate of soil and the divergence of water flow are subjected to the continuity relation. The flow of water is given as $-\mathbf{k} \cdot \nabla p$ (according to Darcy's law) with $\nabla p$ being the pressure gradient.

From the mathematical viewpoint, Eq. (1) is a set of coupling equations of $\mathbf{u}$ and $p$. For simplicity, dropping the rate symbol "." in the first equation, we use the following set of differential equations:

$$
\begin{array}{r}
\rho \mathrm{D}^{2} \mathbf{u}-\nabla \cdot(\mathbf{c}: \nabla \mathbf{u})+\nabla p=\mathbf{0}, \\
\nabla \cdot \mathbf{D} \mathbf{u}-\nabla \cdot(\mathbf{k} \cdot \nabla p)=0,
\end{array}
$$

for stability analysis. Here, superscript $e p$ is omitted for the constitutive tensor $\mathbf{c}^{e p}$.

\section{(2) Stability Analysis}

Soil in general is of heterogeneous nature. For continuum modelling, control volumes of size much larger than solid particles are considered, as shown in Fig. 1. Field variables such as displacement are defined at the scale of control volumes rather than at the scale of particles. Homogeneity is assumed within control volumes for material properties such as elastoplasticity $\mathbf{c}^{e p}$ and permeability $\mathbf{k}$.

We focus on a small homogeneous region. To examine the stability of a solution ( $\mathbf{u}$ and $p$ ) of Eq. (2) in this small region, we consider a fictitious domain, which is extended uniformly to infinity, with the iden- 
tical material properties of the original region. For stability analysis, we assume linear elasticity among this fictitious domain.

In this fictitious domain, we consider the stability of a perturbation, $\delta \mathbf{u}$ and $\delta p$, from a solution $\mathbf{u}$ and $p$ which satisfies the governing equations, Eq. (2). Substitute $\mathbf{u}+\delta \mathbf{u}$ and $p+\delta p$ into Eq. (2) and linearize the equations by omitting terms of $\delta \mathbf{u}$ and $\delta p$ of order higher than 1 . The linearized equation for $\delta \mathbf{u}$ and $\delta p$ is identical to Eq. (2) for a linear elastic tensor c. Hereafter, Eq. (2) is used for the discussion of stability analysis of $\delta \mathbf{u}$ and $\delta p$. Further, $\delta$ is omitted for $\delta \mathbf{u}$ and $\delta p$ for simplicity.

Mathematically, stability and instability are defined for the solutions of the differential equations of dynamic systems ${ }^{12), 13)}$. Consider two initially close states, $\mathbf{x}$ and $\widetilde{\mathbf{x}}$, of a dynamic system,

$$
|\mathbf{x}(0)-\widetilde{\mathbf{x}}(0)|<\delta,
$$

with an arbitrary small value $\delta$. If the deviation between the two states is always bounded for $t>0$,

$$
|\mathbf{x}(t)-\widetilde{\mathbf{x}}(t)|<\epsilon(\forall t>0),
$$

the solutions of the equations for the dynamics system are stable. Otherwise, if the deviation grows as the time increases, especially in an exponential fashion,

$$
|\mathbf{x}(t)-\widetilde{\mathbf{x}}(t)|<C \exp (\lambda t)(\lambda>0),
$$

the solutions are unstable.

In the context of soil dynamics, $\mathrm{x}$ refers to displacement increment $\mathbf{u}$ and water pressure increment $p$. The stability of the solutions of the governing equations is analysed by checking how initial perturbations propagate in a fictitious infinite domain. Exponential increase of perturbations indicates instability.

\section{Plane wave}

In an infinite domain, we analyse the stability of a perturbation as a plane wave which propagates in a fixed direction without changing its form normal to that direction. The plane wave is considered for a pair of $\mathbf{u}$ and $p$. Under the plane wave assumption, we apply the Fourier transform of Eq. (2) using $\exp (\imath(\boldsymbol{\xi}$. $\mathbf{x}-\omega t)$ ), and obtain

$$
\begin{array}{r}
-\rho \omega^{2} \mathbf{u}+(\boldsymbol{\xi} \cdot \mathbf{c} \cdot \boldsymbol{\xi}) \cdot \mathbf{u}+\imath \boldsymbol{\xi} p=\mathbf{0}, \\
\omega \boldsymbol{\xi} \cdot \mathbf{u}+(\boldsymbol{\xi} \cdot \mathbf{k} \cdot \boldsymbol{\xi}) p=0 .
\end{array}
$$

Here, for simplicity, the same symbols $\mathbf{u}$ and $p$ are used for the transformed functions. $\xi$ is the wave vector along which the plane wave propagates and $\omega$ the corresponding frequency.

This set, Eq. (3), is the target equations of the stability analysis for small perturbations in the form of plane wave. We first study a simpler case without considering dilatancy. Then we introduce dilatancy into the stability analysis.

\section{(1) Perturbation without dilatancy}

Without considering dilatancy, the elastic tensor is isotropic, i.e. $\mathbf{c}=\lambda \boldsymbol{\delta} \otimes \boldsymbol{\delta}+2 \mu \mathbf{I}$, where $\boldsymbol{\delta}$ and $\mathbf{I}$ are the second- and (symmetric) fourth-order identity tensor, $\lambda$ and $\mu$ are the Lamé parameters. The permeability is assumed isotropic, i.e. $\mathbf{k}=k \boldsymbol{\delta}$, where $k$ is a scalar permeability parameter. Due to isotropy, for simplicity, we choose a wave vector $\boldsymbol{\xi}=(\xi, 0,0)$ and derive from Eq. (3) the following matrix equation:

$$
\begin{array}{cc}
{\left[\begin{array}{cc}
-\rho \omega^{2}+(\lambda+2 \mu) \xi^{2} & 0 \\
0 & -\rho \omega^{2}+\mu \xi^{2} \\
0 & 0 \\
\omega \xi & 0 \\
0 & \imath \xi \\
0 & 0 \\
-\rho \omega^{2}+\mu \xi^{2} & 0 \\
0 & k \xi^{2}
\end{array}\right]\left[\begin{array}{c}
u_{1} \\
u_{2} \\
u_{3} \\
p
\end{array}\right]=\left[\begin{array}{l}
0 \\
0 \\
0 \\
0
\end{array}\right] .}
\end{array}
$$

The determinant of the matrix at the left-hand side,

$$
D=k \xi^{2}\left(\rho \omega^{2}-\mu \xi^{2}\right)^{2}\left(\rho \omega^{2}-(\lambda+2 \mu) \xi^{2}+\frac{\imath \omega}{k}\right),
$$

must vanish, so that Eq. (4) has non-trivial solutions $\left[u_{1}, u_{2}, u_{3}, p\right]^{T}$.

If $D=0$ is viewed as an equation of $\omega$ for a given $\xi$, we can readily find two roots, $\omega= \pm \xi c_{s}$ with $c_{s}=$ $\sqrt{\frac{\mu}{\rho}}$, which correspond to non-coupling shear waves of $\mathbf{u}$ and $p$. The other two roots of $D=0$ are

$$
\omega=-\frac{1}{2 \rho k}\left(\imath \pm \sqrt{\left(2 \rho k c_{p} \xi\right)^{2}-1}\right)
$$

with $c_{p}=\sqrt{\frac{\lambda+2 \mu}{\rho}}$. These roots are corresponding to coupling plane waves of $\mathbf{u}$ and $p$.

It is interesting to note that $\omega$ in Eq. (5) has a negative imaginary part,

$$
\operatorname{Im}(\omega)=\left\{\begin{array}{l}
-\frac{1}{2 \rho k}, \quad \text { if }\left(2 \rho k c_{p} \xi\right)^{2}>1 \\
-\frac{1 \pm \sqrt{1-\left(2 \rho k c_{p} \xi\right)^{2}}}{2 \rho k}, \text { else }
\end{array}\right.
$$

no matter whether $\left(2 \rho k c_{p} \xi\right)^{2}-1$ is positive or negative. This implies that the coupling plane waves always decay as $t$ increases. Thus, without the dilatancy effect, the solutions of the governing equations, Eq. (2), are always stable and liquefaction would not occur as a transition from stable to unstable solutions.

\section{(2) Perturbation with dilatancy}

A characteristic effect of soil is dilatancy, i.e., shear deformation leads to volumetric deformation. In the present framework of linear stability analysis, dilatancy can be modelled by setting certain components of the elastic tensor c, e.g. $c_{1112}$ or $c_{1113}$, as non-zero. These components actually correspond to the generation of normal stress due to shear strain (or vice versa, 
the generation of shear stress due to normal strain). If the generated normal stress is suppressed by inducing normal strain, then shear strain apparently induces normal strain, which has the same effect as dilatancy.

For simplicity, we set $c_{1112}=c_{1113}=\alpha$ to model the dilatancy effect for stability analysis. Here, $\alpha$ can be regarded a dilatancy modulus, which has SI unit Pa. Replacing the isotropic elastic tensor $\mathbf{c}$ in the non-dilatancy case with this "anisotropic" linear elastic tensor c, we have a matrix equation from Eq. (3):

$$
\left.\begin{array}{cc}
-\rho \omega^{2}+(\lambda+2 \mu) \xi^{2} & \alpha \xi^{2} \\
\alpha \xi^{2} & -\rho \omega^{2}+\mu \xi^{2} \\
\alpha \xi^{2} & 0 \\
\omega \xi & 0 \\
\alpha \xi^{2} & \imath \xi \\
0 & 0 \\
-\rho \omega^{2}+\mu \xi^{2} & 0 \\
0 & k \xi^{2}
\end{array}\right]\left[\begin{array}{c}
u_{1} \\
u_{2} \\
u_{3} \\
p
\end{array}\right]=\left[\begin{array}{l}
0 \\
0 \\
0 \\
0
\end{array}\right] .
$$

Note that the symmetry of the elastic tensor $\mathbf{c}\left(c_{i j k l}=\right.$ $c_{i j l k}=c_{j i k l}=c_{k l i j}$ ) has to be fully considered. The determinant of the matrix at the left-hand side of Eq. (6) becomes

$$
\begin{array}{r}
D=k \xi^{2}\left(-\rho \omega^{2}+\mu \xi^{2}\right)\left(k\left(2 \alpha^{2}+\mu(\lambda+2 \mu)\right) \xi^{4}+\right. \\
\left.\imath \mu \xi^{2} \omega+k(\lambda+3 \mu) \xi^{2} \rho \omega^{2}-\imath \rho \omega^{3}-k \rho \omega^{4}\right) .
\end{array}
$$

For the existence of non-trivial solutions of Eq. (6), $D$ should vanish $(D=0)$, which gives

$$
\begin{gathered}
k \xi^{2}\left(-\rho \omega^{2}+\mu \xi^{2}\right)\left(k\left(2 \alpha^{2}+\mu(\lambda+2 \mu)\right) \xi^{4}+\right. \\
\left.\imath \mu \xi^{2} \omega+k(\lambda+3 \mu) \xi^{2} \rho \omega^{2}-\imath \rho \omega^{3}-k \rho \omega^{4}\right)=0 .(7)
\end{gathered}
$$

The two roots, $\omega= \pm \xi c_{s}$, corresponding to shear waves, are easy to find. Denoting by $\omega_{p, s, a}=c_{p, s, a} \xi$ with $c_{a}=\sqrt{\alpha / \rho}$ and $\omega_{k}=1 / \rho k$, we can simplify Eq. (7) to find the other roots. Furthermore, by introducing non-dimensional variable $r=\omega / \omega_{p}$ and parameters $r_{s, a, k}=\omega_{s, a, k} / \omega_{p}$, we can derive the following characteristic equation:

$$
r^{4}+\imath r_{k} r^{3}-\left(1+r_{s}^{2}\right) r^{2}-\imath r_{k} r_{s}^{2} r+r_{s}^{2}-2 r_{a}^{4}=0 .
$$

By definition, $r$ is the relative velocity as $c_{p}$ is taken as the reference. Note that $r_{s}$ and $r_{a}$ are the relative velocities and independent with the wave number $\xi$, while $r_{k}=\frac{1}{c_{p} k \rho \xi}$ depends on $\xi$.

\section{Existence of unstable solution}

Recall that for a plane wave in the form of $\exp (\imath(\boldsymbol{\xi}$. $\mathbf{x}-\omega t))$ with a complex $\omega$, an $\omega$ with positive imaginary part would indicate an unbounded temporally increase of wave amplitude, i.e. an unstable solution of the governing equations. Thus, the existence of unstable solution depends on the roots of Eq. (8). An $r$ with a positive imaginary part is corresponding to an unstable solution. Closed-form expressions of the roots of Eq. (8) are rather cumbersome. Here we give numerical results of $r$, which are determined by $r_{a}, r_{s}$ and $r_{k}$ explicitly and by $\xi$ implicitly. Note that $r_{s}$ can be represented by Poisson ratio $\nu$ as

$$
r_{s}=\sqrt{\frac{1-2 \nu}{2(1-\nu)}} .
$$

To obtain numerical solutions for $r$, we can set the relative velocity $r_{s}=0.4$ as an example. This $r_{s}$ corresponds to a Poisson ratio $\nu$ about 0.4 , a typical value for soil ${ }^{14)}$. A set of four roots can be determined for the polynomial equation, Eq. (8), for a given pair of $r_{a}$ and $r_{k}$. For $r_{a}$ and $r_{k}$ varying between 0 and 1 , the roots of $r$ are shown on the complex plane as in Fig. 2. As can be seen, there are roots located on the positive imaginary axis. Thus those $r$ located on the positive imaginary axis correspond to unstable solutions of the governing equations.

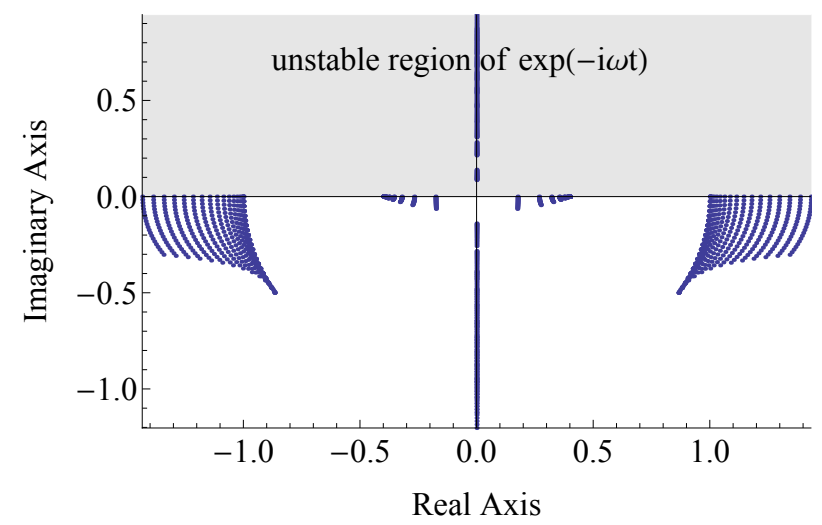

Fig.2 $r$ on the complex plane for a fixed $r_{s}$ as 0.4 and varying $r_{k}$ and $r_{a}$ between 0 and 1 . Those $r$ with positive imaginary part are corresponding to unstable solutions of the governing equations.

\section{Influence of dilatancy ratio $r_{a}$}

The dependence of $r$ on $r_{k}$ and $r_{a}$ for the given $r_{s}$ is shown in Fig. 3. Note that there are four roots for $r$ and we only show the one which are related to unstable solutions. As can be seen, when the dilatancy ratio $r_{a}$ is larger than a critical value $r_{a}^{c}$, this root of $r$ changes from a real number to a positive imaginary number. Physically, this implies that when the dilatancy of soil becomes large, the liquefaction may occur in the sense that the solutions become unstable.

From Fig. 3, we observe that the critical value $r_{a}^{c}$ is virtually constant for the given $r_{s}$. This implies a relation between the critical dilatancy ratio and the relative shear wave velocity $r_{s}$. Assuming a trivial solution of $r=0$ exists for the characteristic equation, Eq. (8), we obtain

$$
r_{s}^{2}-2 r_{a}^{4}=0
$$

This gives $r_{a}$ in terms of $r_{s}$

$$
r_{a}=\left(\frac{r_{s}^{2}}{2}\right)^{\frac{1}{4}}
$$


which turns out to be the critical dilatancy ratio. As presented by the thick lines in Fig. 4, the proposed critical dilatancy ratio is coincident with the transition lines between negative (or zero) and positive imaginary part of $r$.

\section{Influence of permeability}

As discussed above, $r_{k}$, which is proportional to the inverse of the permeability velocity, does not influence the critical dilatancy ratio, beyond which $r$ has positive imaginary part and the corresponding solutions are unstable. To study the influence of permeability on the values of $r$, we extend the range of $r_{k}$ for three cases of $r_{a}$ larger than the critical dilatancy ratio. As shown in Fig. 5, the magnitudes of the imaginary part of $r$ converge to zero as $r_{k}$ increases. This implies that for a very large $r_{k}$, even $r_{a}$ transcends the critical dilatancy ratio, the solutions of the governing equations converge to be stable. The stable solutions can be explained by the extreme situation that the permeability velocity is zero. In this situation, the conservation of mass described in Eq. (2)

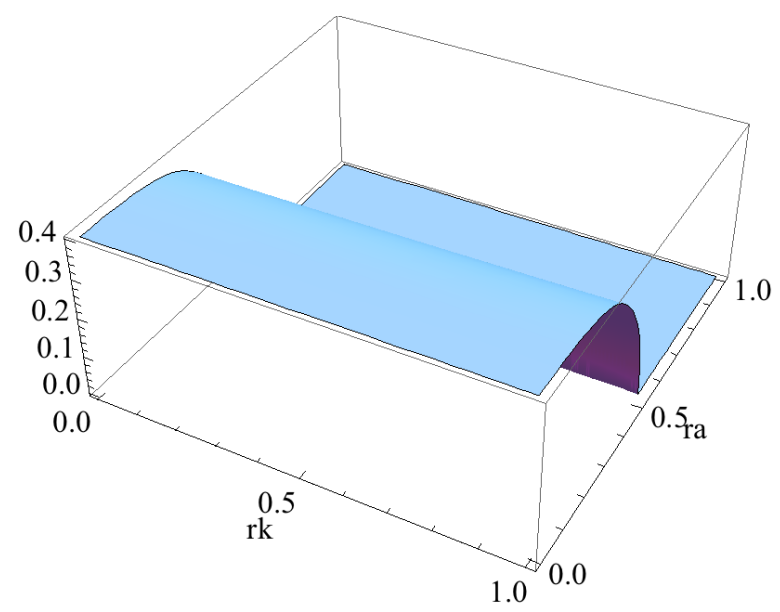

(a) $\operatorname{Re}(r)$

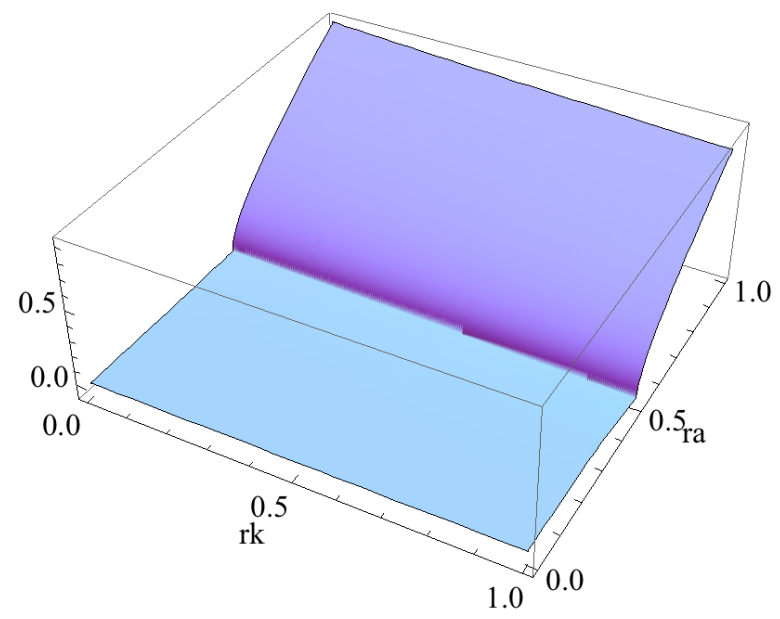

(b) $\operatorname{Im}(r)$

Fig.3 The real part (a) and the imaginary part (b) of $r$ related the unstable solutions of the governing equations for a fixed $r_{s}=0.4$

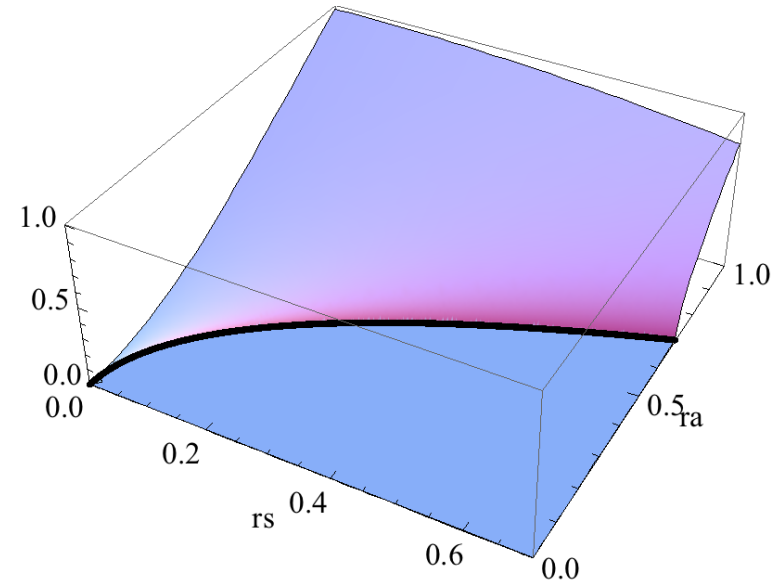

(a) $r_{k}=0.1$

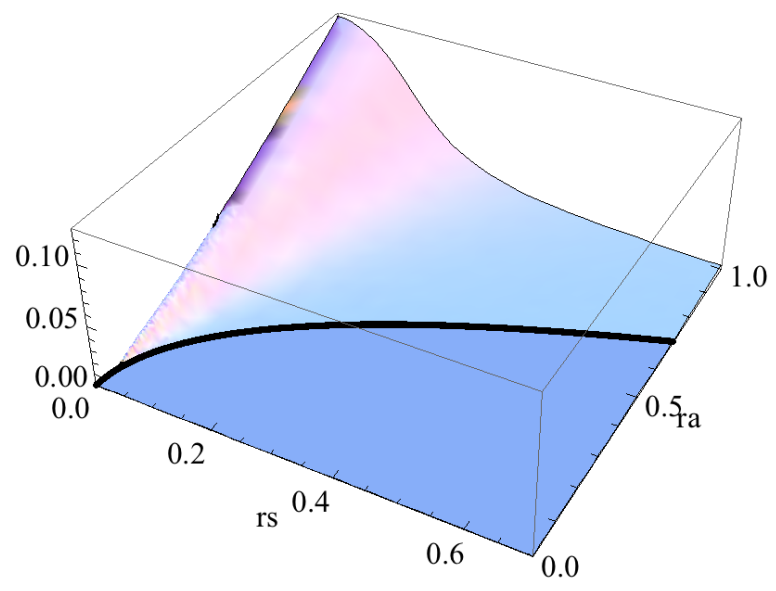

(b) $r_{k}=1000$

Fig.4 Critical dilatancy ratio (thick lines) for the imaginary part of $r$ with fixed $r_{k}=0.1$ (a) and $r_{k}=1000$ (b); Negative imaginary part is truncated as zero.

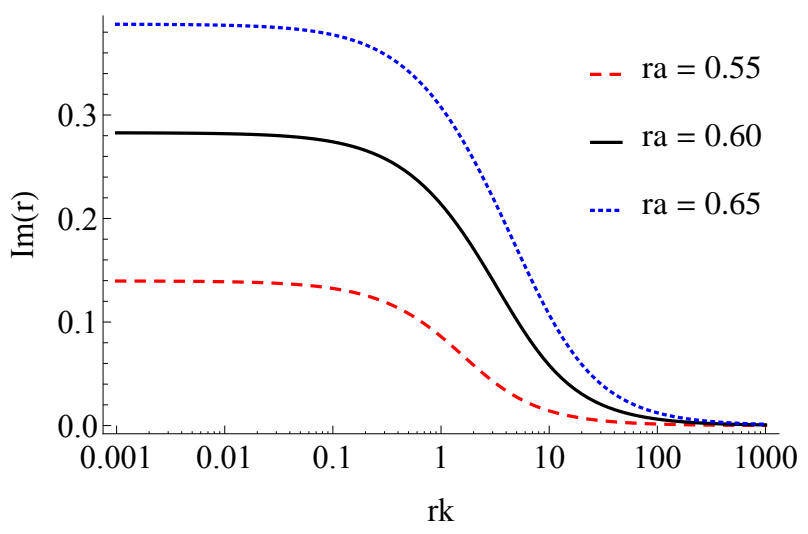

Fig.5 Convergence of the imaginary part of $r$ related to the unstable solutions in a large range of $r_{k}\left(r_{s}=0.4\right)$.

becomes a constraint on volumetric deformation as: $\nabla \cdot \dot{\mathbf{u}}=0$. Note that pure shear waves are valid solutions for the governing equations, Eq. (2), satisfying such constraint. With the increase of $r_{k}$ (decrease of permeability velocity), the unstable solutions are eventually converged to stable shear plane waves. 


\section{Spherical wave}

In this section, we first derive analytical solutions for the stability of perturbations in the form of spherical wave, without considering dilatancy. Then numerical simulations are carried out for dilatancy cases.

\section{(1) Perturbation without dilatancy}

We consider spherical symmetry for perturbations,

$$
\mathbf{u}=u(r, t) \mathbf{e}_{\mathrm{r}}, p=p(r, t),
$$

where $\mathbf{e}_{\mathrm{r}}$ stands for the radical unit vector. For nondilatancy cases, in spherical coordinates system, the governing equations, Eq. (2), become

$$
\begin{aligned}
\mathrm{D}^{2} u-c_{p}^{2}\left(\frac{\partial^{2} u}{\partial r^{2}}+\frac{2}{r} \frac{\partial u}{\partial r}-\frac{2 u}{r^{2}}\right)+\frac{1}{\rho} \frac{\partial p}{\partial r} & =0 \\
\mathrm{D}_{\mathrm{r}}\left(\mathrm{D} u-k \frac{\partial p}{\partial r}\right) & =0
\end{aligned}
$$

with $\mathrm{D}_{\mathrm{r}}=\frac{\partial}{\partial r}+\frac{2}{r}$. Follow the derivation of spherical wave propagation in a pure elastic body ${ }^{15}$, we introduce a displacement potential $\phi$

$$
u=\frac{\partial \phi}{\partial r} \text {. }
$$

Substitute Eq. (14) into the governing equations for spherical wave, we have

$$
\begin{aligned}
\mathrm{D}^{2} \frac{\partial \phi}{\partial r}-c_{p}^{2}\left(\frac{\partial^{2}}{\partial r^{2}}+\frac{2}{r} \frac{\partial}{\partial r}-\frac{2}{r^{2}}\right) \frac{\partial \phi}{\partial r}+\frac{1}{\rho} \frac{\partial p}{\partial r} & =0 \\
\mathrm{D}_{\mathrm{r}}\left(\mathrm{D} \frac{\partial \phi}{\partial r}-k \frac{\partial p}{\partial r}\right) & =0
\end{aligned}
$$

From Eq. (15b), omitting higher order derivatives of $r$, we have

$$
\frac{1}{k} \frac{\partial \mathrm{D} \phi}{\partial r}=\frac{\partial p}{\partial r}
$$

Substitute this into Eq. (15a), we can obtain the following equation of $\phi$

$$
\frac{\partial \mathrm{D}^{2} \phi}{\partial r}-c_{p}^{2}\left(\frac{\partial^{2}}{\partial r^{2}}+\frac{2}{r} \frac{\partial}{\partial r}-\frac{2}{r^{2}}\right) \frac{\partial \phi}{\partial r}+\omega_{k} \frac{\partial \mathrm{D} \phi}{\partial r}=0 .
$$

Integrate this equation once with respect to $r$,

$$
\mathrm{D}^{2} \phi-c_{p}^{2}\left(\frac{\partial^{2}}{\partial r^{2}}+\frac{2}{r} \frac{\partial}{\partial r}\right) \phi+\omega_{k} \mathrm{D} \phi=0 .
$$

Further assumes that $\phi$ takes the form

$$
\phi=\Phi_{0} \frac{\exp (\imath(\xi \cdot r-\omega t))}{r},
$$

where $\Phi_{0}$ is a constant. Substitute this $\phi$ into Eq. (18) we can obtain the following equation for $\omega$,

$$
\omega^{2}+\imath \omega_{k} \omega-c_{p}^{2} \xi^{2}=0 .
$$

The roots of $\omega$ is given as,

$$
\omega=-\imath \frac{\omega_{k}}{2} \pm c_{p} \xi \sqrt{1-\left(\frac{\omega_{k}}{2 c_{p} \xi}\right)^{2}} .
$$

Note that $\omega_{k}$ represents the coupling effect of pore water, serving as damping against elastic waves. Comparing to pure elastic waves (i.e. $\omega_{k}=0$ ), pore water damps out the wave magnitute and slows down the wave velocity. The roots of $\omega$ given in Eq. (21)
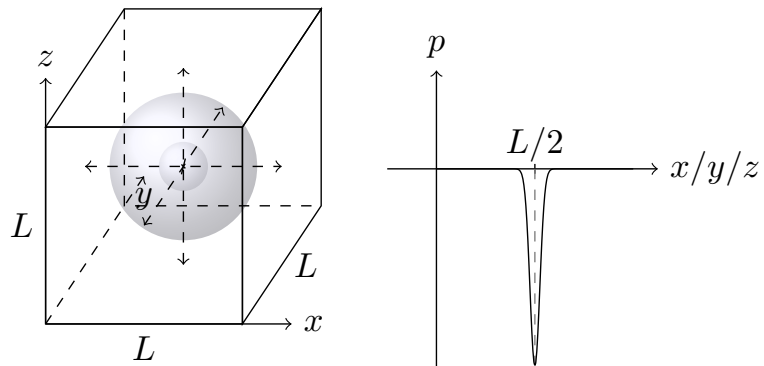

Fig.6 Initial settings for numerical simulation of perturbations in the form of spherical wave: an initial pressure field is prescribed, $p(x, y, z)=-1000$. $\exp \left(-\frac{(x-L / 2)^{2}+(y-L / 2)^{2}+(z-L / 2)^{2}}{2(L / 40)^{2}}\right)$ $\mathrm{Pa}$, which introduces an initial compression with respect to the center of the domain.

are the same as Eq. (5) in the plane wave case without dilatancy. Thus perturbations in the form of spherical wave for non-dilatancy cases are always stable.

\section{(2) Perturbation with dilatancy}

For dilatancy cases, analytical solutions in the frequency domain are difficult to attain for perturbations in the form of spherical wave. Instead, we use a numerical code ${ }^{16)}$ developed for stability analysis of liquefaction, based the particle discretization scheme, which is a finite element method originally proposed for failure problems ${ }^{17), 18)}$. The convergence of the numerical solutions of this code is studied in Ref ${ }^{16}$.

The governing equations are solved numerically in a finite cubic domain for a spherically symmetrical initial perturbation as illustrated in Fig. 6. The material properties in simulation are listed in Table 1. Dilatancy is considered for the $x$-direction $\left(c_{1112}=\right.$ $\left.c_{1113}=\alpha\right)$. The size of the domain is listed in Table 2 , with mesh size as 101 nodes along each axis. In total 1,030,301 nodes and 6,000,000 tetrahedron elements are used for spatial discretization. Such mesh size has been chosen as a trade-off between numerical accuracy and computational cost ${ }^{16)}$.

Table1 Material properties for numerical simulation

\begin{tabular}{ccccc}
\hline $\begin{array}{c}\text { Young's } \\
\text { modulus } \\
E(\mathrm{MPa})\end{array}$ & $\begin{array}{c}\text { Poisson's } \\
\text { ratio } \nu\end{array}$ & $\begin{array}{c}\text { density } \\
\rho\end{array}$ & $\begin{array}{c}\text { permeability } \\
k\end{array}$ & $\begin{array}{c}\text { dilatancy } \\
r_{a}^{2}\end{array}$ \\
\hline 50 & 0.4 & 2000 & $1.02 \cdot 10^{-8}$ & 0.20 \\
50 & 0.4 & 2000 & $1.02 \cdot 10^{-8}$ & 0.30 \\
\hline
\end{tabular}

We consider a perturbation as a deviation from an equilibrium state. Thus it is reasonable to fix the boundary conditions for $\mathbf{u}$ and $p$ as zero. A pressure field is prescribed initially to generate a compressional wave. With the same initial and bound- 

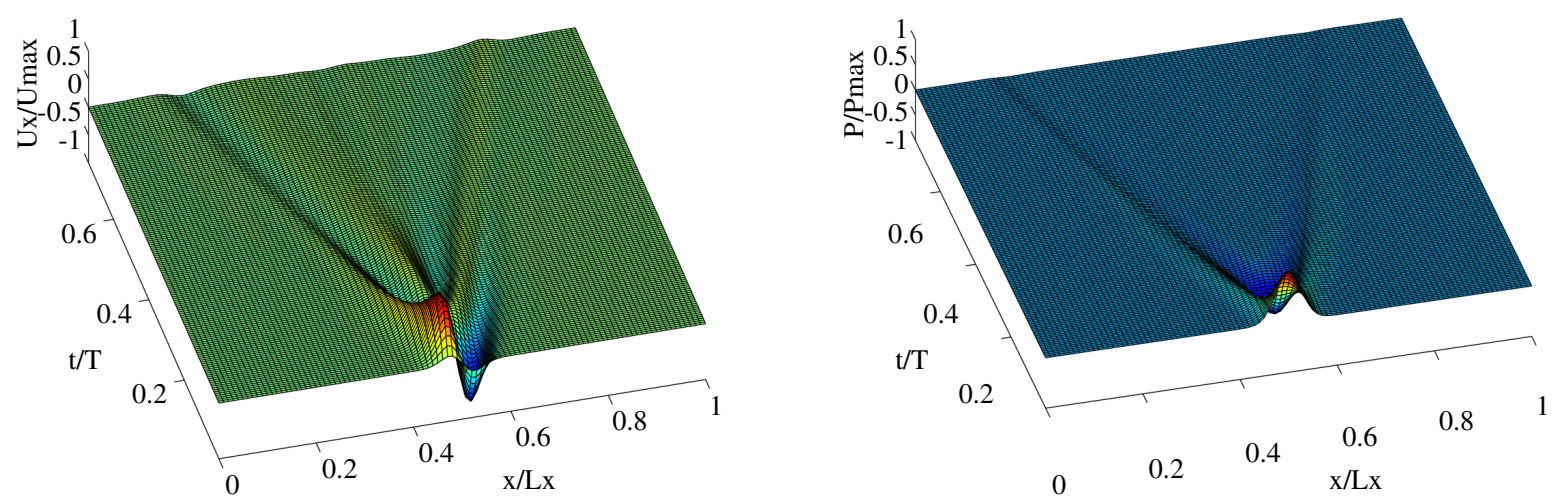

(a) $r_{a}^{2}=0.2$
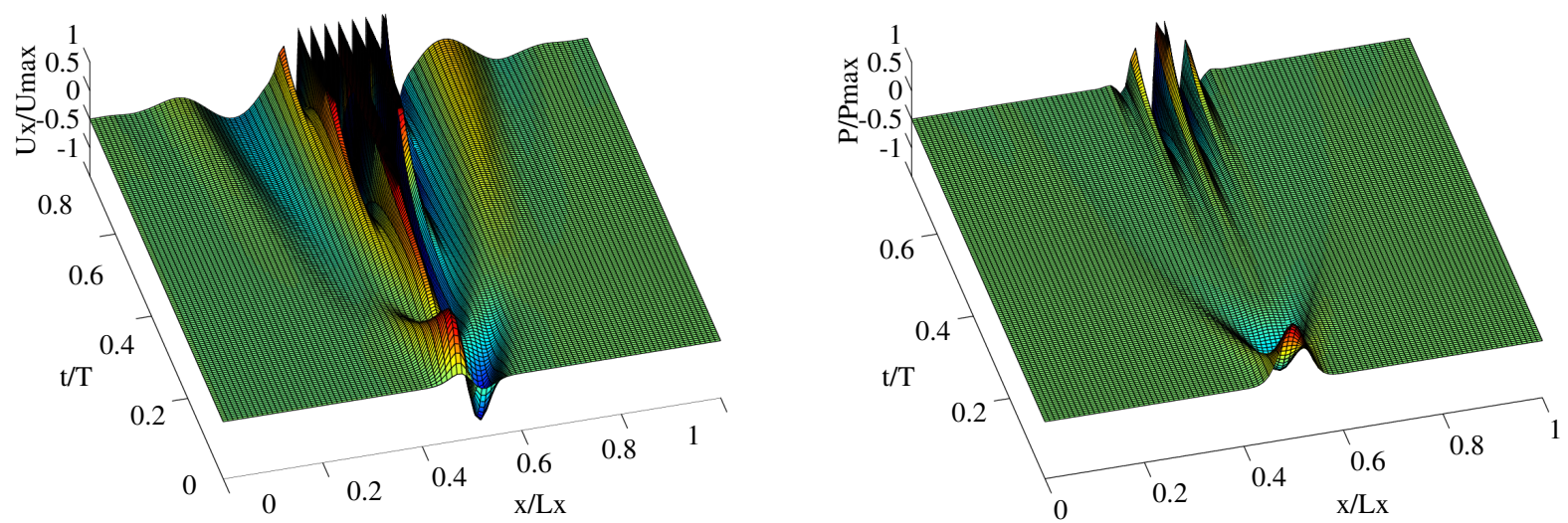

(b) $r_{a}^{2}=0.3$

Fig.7 Simulation results of $\mathbf{u}$ and $p$ at the center line $(y=z=L / 2)$ parallel to the $x$-axis for $r_{a}^{2}=0.2$ and 0.3 . In the case $r_{a}^{2}=0.3$, the unstable solutions larger than the maxima in the stable non-dilatancy case are truncated.

Table2 Configurations for numerical simulation

\begin{tabular}{cccc}
\hline length & primary wave & reference time & time-step \\
$L(\mathrm{~m})$ & velocity $c_{p}(\mathrm{~m} / \mathrm{s})$ & $T=\frac{L}{2 c_{p}}(\mathrm{~ms})$ & size $\delta t / T$ \\
\hline $1 \cdot 10^{-3}$ & 231.46 & $2.16 \cdot 10^{-3}$ & $4.63 \cdot 10^{-3}$ \\
\hline
\end{tabular}

ary conditions, the maximum displacement increment $\mathrm{U}_{\max }$ and pressure increment $\mathrm{P}_{\max }$ in the stable nondilatancy case are measured and used to normalize the solutions of $\mathbf{u}$ and $p$ in dilatancy cases. The time for a wave with velocity $c_{p}$ propagates from the center to the boundary of the domain is taken as the reference time, see Table 2.

The results of $\mathbf{u}$ and $p$ along the center line $(y=$ $z=L / 2$ ) are shown in Fig. 7 for two cases of different dilatancy ratio $\left(r_{a}^{2}=0.2\right.$ and $\left.r_{a}^{2}=0.3\right)$. For the small dilatancy case $\left(r_{a}^{2}=0.2\right)$, the prescribed perturbations propagate stably with decaying amplitude, from the center region to the boundaries of the domain. For the large dilatancy case $\left(r_{a}^{2}=0.3\right)$, the un- stable solutions grow exponentially within a distinct region around the center. For spherical perturbations, the existence of unstable solutions is confirmed.

The first stress invariant $I_{1}\left(I_{1}=\sigma_{k k}\right)$ and the second deviatoric stress invariant $J_{2}\left(J_{2}=\sigma_{i j} \sigma_{i j} / 2-\right.$ $\left.\left(\sigma_{k k}\right)^{2} / 6\right)$ are computed. The maximal first stress invariant for the non-dilatancy case (i.e. with an "isotropic" elastic tensor) under the same simulation settings, $\mathrm{I}_{\max }^{\text {iso }}$, are used for normalization. The (averaged) stress invariants around the central planes $x=$ $L / 2$ and $z=L / 2$ at $t / T=0.74$ in the large dilatancy case are shown in Fig. 8. The results for $y=L / 2$ are omitted considering the symmetry of $y$ and $z$ in the setting of dilatancy. As can be seen, as the consequence of the unstable solutions of displacement, the stresses around the center region are accumulated. Further investigations are needed to clarify the possibility of the propagation of unstable solutions considering the formation of "micro cracks", weakening of soil strength when the accumulated stresses exceed a certain yield criterion. 

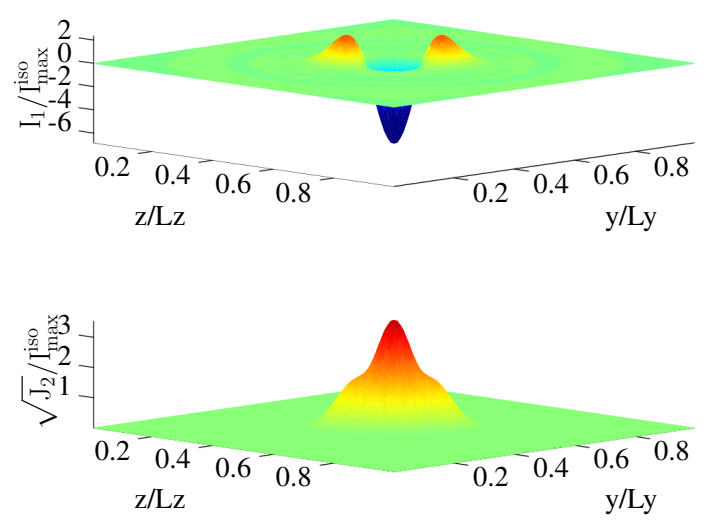

(a) $x=L / 2$
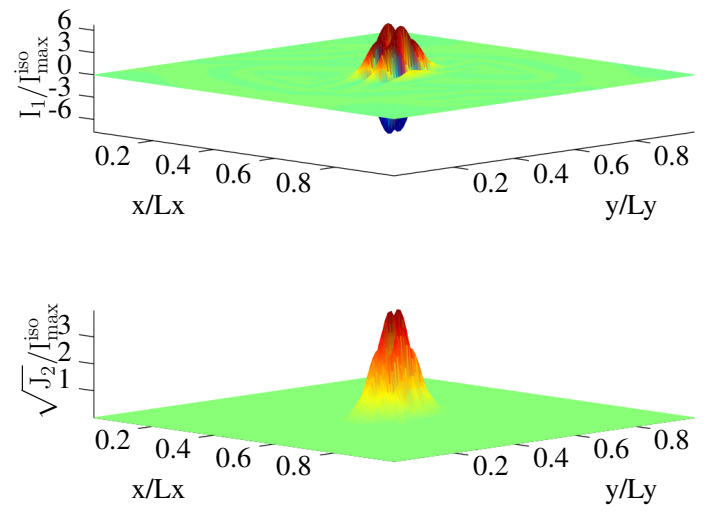

(b) $z=L / 2$

Fig.8 Stress invariants at the central planes (a) $x=L / 2$ and (b) $z=L / 2$ at time $\mathrm{t} / \mathrm{T}=0.74$.

\section{Conclusions}

In this paper we studied the stability of the solutions of the governing equations of soil liquefaction, with perturbations in the form of plane wave and of spherical wave. Dilatancy is modelled by setting some entries of the elastic tensor as non-zero. For perturbations in both forms, without dilatancy, the solutions are always stable thus no possibility of liquefaction. In the presence of dilatancy, the stability is lost when the dilatancy ratio exceeds a critical value. The critical dilatancy ratio has been derived for the plane wave case. For spherical perturbations, numerical simulations showed similar influence of dilatancy ratio on the stability of the solutions. The loss of stability indicates a possible initiation of liquefaction. Stability analysis as done in this paper provides a new perspective on the initiation of liquefaction, as a possible transition from stable to unstable solutions. As shown in the numerical simulations, the unstable solutions seems to be confined within the central region. To investigate the possibility of propagation of unstable perturbations will be a future study.

\section{REFERENCES}

1) Ishihara, K.: Liquefaction and Flow Failure During Earthquake, Gétechnique, Vol.43, pp.351-415, 1993.

2) Kramer, S.: Geotechnical Earthquake Engineering, Prentice Hall, 1996.

3) Seed, R. B., Cetin, K., Moss, R., Kammerer, A., Wu, J., Pestana, J., Riemer, M., Sancio, R., Bray, J., Kayen, R., and Faris, A.: Recent Advances in Soil Liquefaction Engineering: A Unified and Consistent Framework, 26th Annual ASCE Los Angeles Geotechnical Spring Seminar, 2003.

4) Zeghal, M. and El Shamy, U.: A Continuum-Discrete Hydromechanical Analysis of Granular Deposit Liquefaction, Int. J. Numer. Anal. Meth. Geomech., Vol.28, pp.1361-1383, 2004.

5) Hakuno, M. and Tarumi, Y.: Sand Liquefaction analysis by granular assembly simulation, Proceedings of Ninth World Conference on Earthquake Engineering, pp. VIII-231 - VIII236, August 2-9 1988.

6) Biot, M.: Theory of Propagation of Elastic Wave in A Fluid-Saturated Porous Solid, Journal of the Acoustical Society of America, Vol.28, pp.168-178, 1956.

7) Zienkiewicz, O., Chan, A., Pastor, M., Schrefler, B., and Shiomi, T.: Computational Geomechanics, Wiley, 1999.

8) Gudehus, G.: Physical Soil Mechanics, Springer, 2011.

9) Oka, F. and Kimoto, S.: Computational Modelling of Multiphase Geomaterials, CRC, 2012.

10) Wong, Y. and Pellegrino, S.: Wrinkled Membranes Part III: Numerical Simulations, Gétechnique, Vol.43, pp.351-415, 1993.

11) Sasiharan, N.: Mechanics of Dilatancy and Its Application to Liquefaction Problems, PhD thesis, Washington State University, 2006.

12) Seydel, R.: Practical Bifurcation and Stability Analysis, Springer, third edition edition, 2010.

13) Matuttis, H.-G. and Chen, J.: Understanding the Discrete Element Method: Simulation of Non-Spherical Particles for Granular and Multi-body Systems, Wiley, 2014.

14) Knappett, J. and Craig, R.: Craig's Soil Mechanics, Spon Press, eighth edition, 2012.

15) Verruijt, A.: An Introduction to Soil Dynamics, Springer, 2010.

16) Chen, J., O-tani, H., and Hori, M.: Stability Analysis for Local Liquefaction Initiation of Plane Wave Type, Proceedings of the 14th International Conference of the International Association for Computer Methods and Advances in Geomechanics, pp. 661-666, 2014.

17) Hori, M., Oguni, K., and Sakaguchi, H.: Proposal of FEM Implemented with Particle Discretization for Analysis of Failure Phenomena, J. Mech. Phys. Solids, Vol.53, pp.681-703, 2005.

18) Wijerathne, M., Oguni, K., and Hori, M.: Numerical Analysis of Growing Crack Problems using Particle Discretization Scheme, Int. J. Numer. Meth. Engng., Vol.80, pp.46-73, 2009.

(Received June 20, 2014) 